1

2

3

4

5

6

7

\title{
An Evacuation Model Validation Data-Set for High-Rise Construction Sites
}

\author{
Steven Deere ${ }^{\mathrm{a}}$, Xie Hui ${ }^{\mathrm{b}}$, Edwin R. Galea ${ }^{\mathrm{c}^{*}}$, David Cooney ${ }^{\mathrm{d}}$, Peter J. Lawrence ${ }^{\mathrm{e}}$
}

a University of Greenwich, Park Row, Greenwich, London, UK, s.deere@gre.ac.uk

bUniversity of Greenwich, Park Row, Greenwich, London, UK, h.xie@gre.ac.uk

'University of Greenwich, Park Row, Greenwich, London, UK, e.r.galea@gre.ac.uk

dUniversity of Greenwich, Park Row, Greenwich, London, UK, d.p.cooney@gre.ac.uk

eUniversity of Greenwich, Park Row, Greenwich, London, UK, p.j.lawrence@gre.ac.uk

*Corresponding author

\section{Highlights:}

- A unique high-rise construction site evacuation validation data-set is presented.

- A metric is presented to measure performance of evacuation simulation software.

- The data-set and metric are used to assess the performance of buildingEXODUS.

\begin{abstract}
:
Evacuation of high-rise construction sites is one of the most challenging evacuation scenarios conceivable. Over the past 50 years, very little evacuation research has focused on issues uniquely associated with high-rise construction sites. To address this, FSEG, in collaboration with IOSH and Multiplex, undertook a three-year project to develop an evidence base describing evacuation performance of high-rise construction site workers. This data was used to define a unique evacuation validation data-set for high-rise construction sites. The validation data-set, described in this paper, contains a complete description of the evacuation scenario, including geometry, population, procedures, response times and exit curves. A performance metric is defined which objectively describes the goodness of fit between model predictions and experimental data. Given the level of uncertainty in the validation data-set an objective measure of acceptable agreement between the model prediction and the experimental data is specified for the metric. The level of acceptability is based on the performance of a modified version of buildingEXODUS, which provides a benchmark defining an acceptable level of agreement with the experimental data. The analysis demonstrates that suitably adapted evacuation simulation software can predict the evacuation performance of high-rise construction sites with a reasonable level of accuracy.
\end{abstract}

Keywords: modelling; human behaviour; response times; evacuation; high-rise building; construction sites; validation data-set

\section{Introduction}


The skyline of modem cities continues to grow upwards driven by the demand for more residential and commercial space on finite available land resources. The soaring scale of high-rise building construction - the number of projects and the size of the buildings - is reflected in the number of workers exposed to these demanding construction environments, and the potential for large-scale evacuation. In London alone, an estimated 541 high-rise building projects are planned for the next few years [1]. Construction sites represent a significant fire risk due to the nature of some work being carrying out on site (e.g. hot work), the storage of flammable materials and often the lack of fully operational fire detection and protection measures. Although fatal fires on UK construction sites have been rare in recent years [2], there are still several thousand construction site fires annually in the UK [3].

Evacuation during the construction phase of a high-rise building is one of the most challenging evacuation scenarios. Due to the dynamic nature of the construction environment in terms of building layout, tasks and workforce, there are several inherent challenges to safe evacuation. Firstly, fire detection and protection measures intended to operate in the completed building may not be in place or fully operational. Thus, evacuation routes are less likely to offer a safe refuge in the event of fire, and may involve temporary surfaces such as decking and decking with rebar, while connections between floors may involve narrow and steep temporary scaffold stairs or ladders. These types of terrain are likely to have a negative impact on evacuation performance. Secondly, the very layout of the building, the interconnectivity and traversability of paths, are constantly changing during construction making it more difficult for the constantly changing workforce to wayfind. Evacuation plans and procedures must adapt to the changing physical environment and workforce makeup. These plans must be conveyed to and understood by the workforce, a workforce made up of people from many different nationalities who may not be fluent native language speakers [4]. Thirdly, in the event of an emergency, the manner in which the population is alerted to the need to evacuate may vary due to the availability of power for alarm systems, the level of noise and the nature of ear protection worn by workers. Some activities may require workers to make safe a pre-alarm activity, thus delaying their response. As a result, the response times for construction workers may vary widely and not follow the typical 'log-normal' distribution commonly found in other evacuation environments [5], [6]. Finally, regular evacuation drills [3], [7] will be required to prepare the workforce for an emergency and as a means to better understand the potential issues and optimise evacuation plans and procedures. However, these are seldom unannounced and often fail to test workers' knowledge of the evacuation process, their responses, and the effectiveness of the procedures in place and the training processes employed.

These unique issues make evacuation of construction sites one of the most challenging evacuation scenarios conceivable. While some research has been conducted concerning construction site safety in the past [8]-[11], globally over the past 50 years, very little, if any evacuation research has focused on the issues uniquely associated with high-rise construction sites.

One of the few studies with a specific focus on evacuation behaviour of construction workers in tunnels was undertaken by Lund University [8]. The work is important as it highlights some of the unique issues associated with the evacuation of construction sites and the general lack of an evidence base to support both regulation and modelling. However, the data collected is very limited and only applicable to very specific tunnel applications. Hisham et al. [9] proposed developing and using an agent-based evacuation model based on the social forces concept to simulate construction site evacuation. However, they fail to identify many challenging issues associated with construction site evacuation and how these would be addressed by such a model. 
Indeed, they even claim that their model would assume that all the workers on site would be totally familiar with the (changing) nature of the construction site. They also fail to identify the need for an evidence base to calibrate evacuation models or how the evacuation model would be verified or validated. Abune'meh et al. [10] developed a spatial layout model using the space syntax approach to identify the optimal layout for a construction site in order to minimise the risk associated with potential incidents occurring. However, this approach does not automatically identify optimal evacuation paths nor take into account the impact of developing hazard and the behaviour and performance of the workers during an evacuation.

Leite et al. [11] published a paper identifying the modelling, simulation and visualisation grand challenges facing the construction industry. Through a workshop and survey, they identify the incorporation of human behaviour into agent based simulations models as the fourth most challenging grand challenge for the construction industry, with verification and validation of simulation models being the third. The survey further found that the experts agreed that most of the challenges associated with verification and validation could be addressed through the collection of data from construction sites.

In addition to research, Health and Safety (HS) authorities impose regulations and guidelines to reduce fire risks and ensure safety of workers on construction sites. In the UK for example, codes for fire safety and safety practice have been produced by the Loss Prevention Council (LPC) to minimise insured risks [7]. The Regulatory Reform (Fire Safety) Order 2005 [12] and the Management of Health, Safety and Welfare Regulations 1999 [13] require an assessment-based approach to controlling risks associated with fire and other emergencies. General guidance on evacuation safety for construction sites comes under the remit of HS with specific guidance contained within the Health and Safety Executive (HSE) publication HSG 168 [3].

Furthermore, evacuation simulation tools [14], [15] could also be used to assist HS managers to develop, plan and test evacuation procedures for various construction phases. Over the years, a considerable database of human performance and behaviour has been established to calibrate these models so that they can be used reliably [16]. Validation data-sets have been developed to assess how accurately evacuation models can predict evacuation performance thereby providing regulators, designers and building operators with confidence in the results produced using these models [16], [17].

However, it is not clear if these models and the data describing the human behaviour and performance are appropriate to address the identified challenges of high-rise construction sites. For example, how quickly do workers react to the evacuation alarm, how quickly can they walk over the various surface types, how is wayfinding impacted by on-site conditions, etc. This data is essential to frame meaningful regulations and guidance to calibrate evacuation models thereby enabling them to be used for construction site applications. Furthermore, to improve the confidence in applying evacuation models to construction site applications it is necessary to develop a range of reliable validation data-sets representative of construction site evacuation scenarios.

In order to address these issues, Fire Safety Engineering Group (FSEG) at the University of Greenwich in collaboration with the Institution of Occupational Safety and Health (IOSH) and Multiplex Europe undertook a three-year project which involved four full-scale unannounced evacuation trials conducted at two high rise buildings at two different heights of construction, and five walking speed experiments. In total 1078 participants were involved in the nine trials, 
generating a data-set of around 2200 data points [18]. The project developed a unique evidence base characterising, for the first time, the actual performance and behaviour of construction workers during emergency evacuation. The evidence base consists of (i) response times for workers, (ii) worker walking speeds on different types of surfaces, and (iii) worker ascent and descent speeds on temporary dogleg and parallel scaffold stairs and ladders. The data for (i) was derived from the four full-scale evacuation trials while the data for (ii) and (iii) were derived from the five separate and unconnected walking speed trials. The data from (ii) and (iii) has been incorporated in the building evacuation simulation tool buildingEXODUS [14], [15], providing it with a unique capability to simulate evacuation from high-rise construction sites. A complete report describing this work and the data was published by IOSH in September 2019 [18]. In this paper we focus on the High-Rise Construction Site Validation Data-Set (HRCSVDS), based on one of the four full-scale trials, the metric developed to assess model performance and demonstrate its application to the buildingEXODUS software. All data required to define the HRCSVDS and tools to undertake the assessment can be found on the FSEG web site at [19] and so will only briefly be described in this paper.

\section{The challenging physical environment associated with high-rise construction site evacuation}

Most high-rise construction sites consists essentially of two above ground regions, the main part of the building and the formworks. The main part of the building consists of the core and completed or partially completed floors. The partially completed floors can consist of concrete floors, floors consisting of decking and decking and rebar. The floor space can be cluttered with building materials, equipment and scaffolding making it difficult to navigate around the floor. Furthermore, protective netting may be used to close off parts of the floor for safety reasons. The floors can be connected by completed regular stairs, temporary scaffold stairs (dogleg and parallel in configuration) or ladders. The core is built using a climbing formwork i.e. a slipform or a jumpform. The formwork is a cramped and often crowded space, containing shutters which act as a mould into which concrete is poured. A slipform is continuously (and slowly) moving upwards whereas in the case of a jumpform, the core will be built a level at a time and then the form will 'jump' to the top ready for the construction of the next level.

The above ground high-rise construction building population is essentially split into two subpopulations, those workers located in the formworks and those workers located in the main part of the building. During an evacuation workers located in the formworks will have to descend down to the main part of the building, possibly using ladders, and continue down to the ground using the temporary and permanent stairs. Workers located in the main part of the building may have to walk over temporary floor surfaces to a temporary stair or ladder, descending several floors before transferring to a permanent stair as they make their way down to the ground.

\section{The validation data-set}

An evacuation validation data-set can be used to gauge the ability of an evacuation software tool to represent the particular behaviour and evacuation performance for a particular scenario. The validation data-set presented in this paper is intended to represent the specific features and issues 
associated with high rise construction sites. These include geometric specific features (e.g. floor surface types, nature of temporary stairs and ladders, etc.), procedural specific issues (e.g. restricting flow on temporary stairs), population specific issues (e.g. initial population distribution) and behavioural specific issues (e.g. response time distributions). Defining such a validation dataset also requires data defining the initial conditions of the evacuation trial, including an accurate description of the geometry, the total number of people within the building, their initial distribution and representative response times. Finally, the data-set should also include the exit curve (or curves if several key monitoring locations are identified) for the trial.

Once these specific features, issues and initial conditions are specified, the ability of the software tool to accurately and reliably reproduce the evacuation scenario can be gauged by comparing the model predictions with the results from the trial. The comparisons are not restricted to the total evacuation time, but also evacuation curves generated at key locations within the building, the most important being the main exit curve. Other key locations can also be considered; for construction sites, this may include the formworks.

Whether or not the model predictions are considered to be a good representation of the experimental data must be determined in an objective way. This is achieved by defining a performance metric which measures the level of agreement between the model predictions and the experimental data. However, the level of acceptability must take into consideration any uncertainties that may exist within the validation data-set, such as the starting location and the response times of the workers, and the specific paths taken by them.

Of the four unannounced full-scale trials conducted, Trial 4 provided the most complete data-set for consideration of creating an appropriate HRCSVDS. The construction site at 22 BG essentially had one vertical exit route from each floor greatly simplifying the process of collecting sufficient accurate data to define a validation data-set. At the time of Trial 4, 22 BG extended over 39 levels in the South Core and 33 levels in the North Core with 20 levels in the process of being constructed.

At the time of the trial, only the North Core was sufficiently populated to allow the specification of a meaningful data-set. Although there were insufficient number of cameras to cover all of the floors, there were sufficient to obtain a reasonable level of granularity in terms of starting floor for workers. During Trial 4, 382 workers were evacuated. The details of the validation data-set based on the data collected from Trial 4 are briefly presented in the following sections, readers are referred to [18] and [19] for details.

\subsection{Geometry}

The geometry for the validation data-set is based on the North Core of $22 \mathrm{BG}$, which has 33 levels above the ground at the time of Trial 4. Of these, Level 1 to Level 20 are complete or partially complete levels, while Level 21 to Level 32 are core only levels with the jumpform at Level 33. These levels have four types of floor surface: concrete from Level 1 to Level 14, decking with rebar from Level 15 to Level 16, decking from Level 17 to Level 20, and partial decking from Level 21 to Level 22 (steel framework with some decking on).

The vertical means of egress consists of permanent stairs from the ground floor up to Level 8 , temporary stairs from Level 8 up to Level 28, hanging stairs from Level 28 up to the jumpform and ladders within the jumpform. The temporary dogleg staircase is internal to the building from 
Level 8 to Level 18 and external from Level 18 to Level 28. The dimensions of all three staircases, including landing dimensions and the dimensions of the ladders can be found in the HRCSVDS [19].

The jumpform has two main decks, a bottom deck and a top deck, with a hanging deck located between the two. The exit from the jumpform is via the hanging stairs, while the only entrance to the hanging stairs is on the top deck. Workers on the bottom deck have to climb up one of two ladders to reach the top deck and then the hanging stairs. On all the other levels, workers could enter the external and internal temporary stairs and the permanent stairs directly from their floors.

\subsection{Population}

The population for the validation analysis consists of 382 workers evacuated from the construction site of 22 BG in Trial 4. The population consisted of predominately males with an age range of approximately 18 to 65 years old. As there were insufficient number of cameras to completely cover every floor in the trial, the estimation of starting position is limited to the number of workers on each floor where there was a camera at that level, or a range of floors where there was a camera at the lower level. This introduces two uncertainties in determining the distribution of the population in the geometry. First, the precise starting location of each worker on a floor (or a deck in the jumpform) is unknown. Second, for some workers their starting location is only known to be within a specified range of floors. There are 75 workers located between Levels 4 and 6, 49 workers located between levels 9 and 17 and 3 workers located between Levels 28 and 32. Thus, in total, the location of 127 workers in the main building is not known precisely. Despite the uncertainties, it was identified with certainty that 37 workers were located in the jumpform and 345 were located on the other 32 levels of the building (13 level groups).

Located on Level 3 were staff canteen, offices, lockers and changing rooms. The activities at these facilities were not recorded and so the response times of the workers on Level 3 and below were not included in the validation data-set. As a result, the validation data-set is restricted to the 277 workers located above Level 3, which include the 37 workers initially located in the jumpform and the 190 workers initially located in the main building from Level 4 to Level 32 . Note that although the 155 workers initially located on Level 1 to Level 3 were excluded from the validation data-set, their presence may cause congestion at the stairs on Level 3, effectively delaying the 'exit' of the workers descending from higher up in the trial. Therefore, these workers were included in the setup of the simulation model used for the validation analysis, but their exit times are not included in the analysis.

\subsection{Evacuation procedures}

Once workers descended down the hanging stairs from the formworks and walked across to the top of the temporary external stairs on Level 28 , a supervisor regulated the flow of workers onto the stairs. This is understood not to be a normal procedure on the building site but was an ad hoc procedure implemented by a supervisor. The supervisor allowed approximately six workers to enter the temporary stairs approximately every $60 \mathrm{~s}$. This procedure must be represented within 
the validation case. As part of worker induction, they are trained to evacuate immediately on hearing the alarm and it is emphasised that they must abandon their tasks and exit without running.

\subsection{Response times}

Given the differences in the nature of the physical space and activities undertaken in the two regions, it was necessary to investigate the response behaviour of the workers in the two regions separately. The response time settings for the validation data-set consists of two response time distributions, one for workers in the jumpform (see Fig. 1) and the other for workers in the main building (see Fig. 2). The response times used in the validation analysis are not those derived from the specific trial defining the validation data-set. They are the generalised response time distributions appropriate for construction sites derived from data collected from the four full-scale unannounced evacuation trials [18]. The response times for workers in the jumpform is represented by a normal distribution while the response time for workers in the main building is represented by a log-normal distribution.

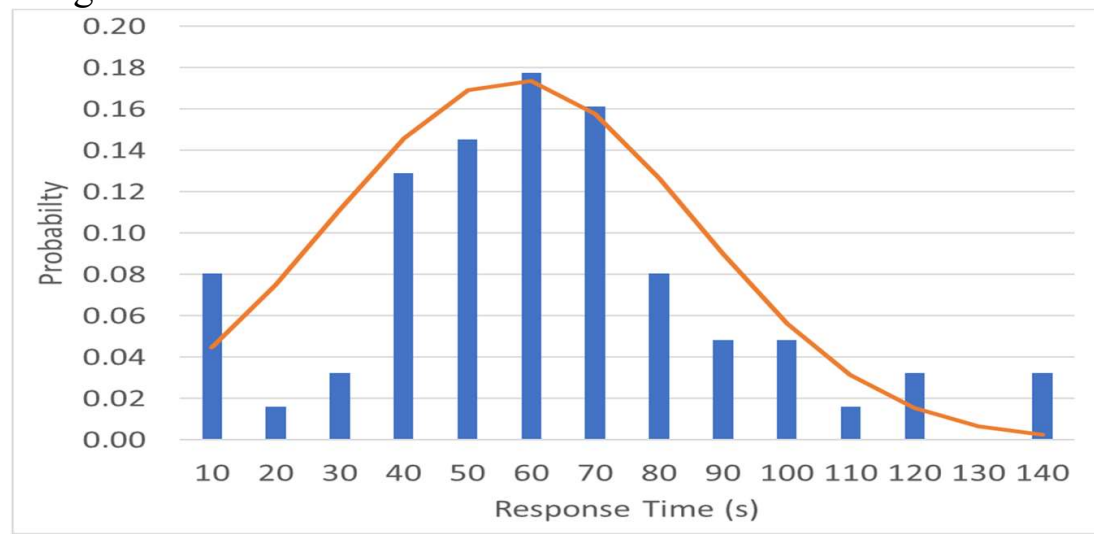

Fig. 1. Generalised response time distribution for workers in the jumpform.

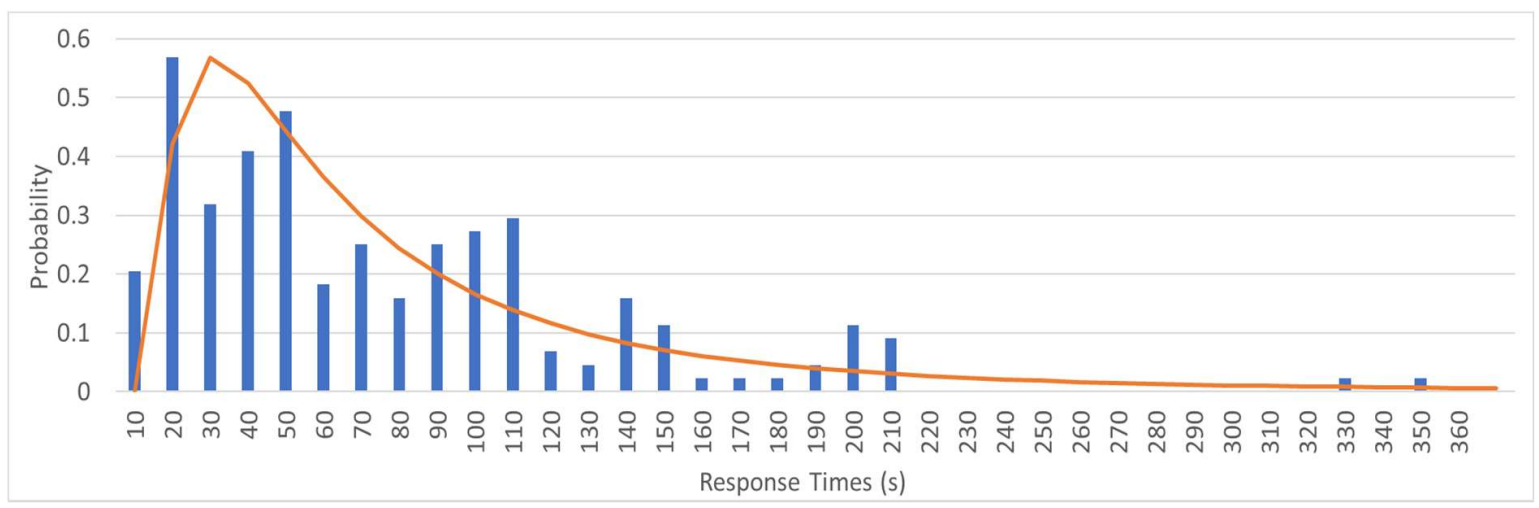

Fig. 2. Generalised response time distribution for workers in the main building.

\subsection{Exit curves}

Two exit curves derived from Trial 4 are used in the HRCSVDS to represent the evacuation performance of $22 \mathrm{BG}$. They are the main exit curve for the entire population above Level 3 (see 
Fig. 3) and the jumpform exit curve for the sub-population in the jumpform (see Fig. 4). The end point for the validation analysis of the main building is the bottom of the flight of permanent stairs that ends on Level 3. The evacuation time for each worker is defined as the time at which the worker's trailing leg leaves the last tread of the stairs. The end point for the validation analysis of the jumpform is the entrance to the hanging stairs on the top deck. The jumpform evacuation time for each worker is defined as the time at which the worker first steps onto the top step of the hanging stairs. Differences in appearance between the two curves can be explained by the nature of the vertical means of escape available and the occupant distribution.


Fig. 3. Main exit curve for the entire population located above Level 3.
Fig. 4. Jumpform exit curve for workers in the jumpform.

\section{Performance metric}

Along with the HRCSVDS it is desirable to specify objective performance measures of the level of agreement between the predicted data and the measured data rather than simply rely on subjective assessments. This is particularly important if the validation analysis is to be used by regulatory authorities to determine the suitability of an evacuation modelling tool. Several approaches are available to measure the level of agreement [17], [20], [21] however, given the nature of the data available the most appropriate is based on [17], [20]. Based on the work of Peacock et al. [20], the authors defined a performance metric that could be used to assess how well evacuation model predictions agreed with a set of experimentally based validation evacuation curves [17]. It is noted that the equations defining the metric in [20] were incorrect and were corrected in [17]. Furthermore, while the methodology in [17] was used for maritime evacuation models, it is suggested that the methodology is equally applicable to building evacuation models, albeit with a different set of acceptance criteria. Details of the performance metric can be found in [17], here we simply present the equations defining the metric.

The series of measured experimental data is represented by an $n$-dimensional vector $E=\left(E_{1}, E_{2}\right.$, .... $E_{n}$ ), where $E_{i}$ represents the measured assembly time for the $i^{\text {th }}$ person. Similarly, the series of predicted model data is represented by vector $\mathrm{m}=\left(\mathrm{m}_{1}, \mathrm{~m}_{2}, \ldots . \mathrm{m}_{\mathrm{n}}\right)$, where $\mathrm{m}_{\mathrm{i}}$ represents the predicted evacuation time for the $i^{\text {th }}$ agent. The metric used to quantify the level of agreement between the predicted and measured values consists of three measures. The first is the Euclidean Relative Difference (ERD) defined by Eq. 1. This is used to assess the average difference between the experimental data $\left(\mathrm{E}_{\mathrm{i}}\right)$ and the model data $\left(\mathrm{m}_{\mathrm{i}}\right)$, i.e. the overall agreement between the two curves. This equation should return 0 if the two curves are identical in magnitude. The second 
measure is the Euclidean Projection Coefficient (EPC) defined by Eq. 2. The EPC calculates a factor which, when multiplied by each model data point $\left(\mathrm{m}_{\mathrm{i}}\right)$, reduces the distance between the model (m) and experimental (E) vectors to its minimum. Thus, the EPC provides a measure of the best possible level of agreement between the model (m) and experimental (E) curves. An EPC of 1.0 suggests that the difference between the model $(\mathrm{m})$ and experimental $(\mathrm{E})$ vectors are as small as possible. The third measure is the Secant Cosine (SC) defined by Eq. 3. Unlike the other two measures, it provides a measure of how well the shape of the model data curve matches that of the experimental data curve. It makes use of the secants (which approximate to tangents) through both curves. An SC of 1.0 suggests that the shape of the model $(\mathrm{m})$ curve is identical to that of the experimental (E) curve.

In Eq. $3 t$ is a measure of the spacing of the data. For the evacuation data representing the exit curves the spacing of the data is 1, i.e. there is a data point for each worker/agent that exits the building. In Eq. $3 s$ is a factor that represents the period of noise in the data, or variations in the experimental data resulting from microscopic behaviour not possible to reproduce in the model. Selecting a value of $s$, which is greater than the period of the noise in the data, provides a means to smooth out the effect of the noise.

In general, for the model and experimental curves to be considered a perfect match, it is necessary to have all three measures at their optimal values, i.e. $\mathrm{ERD}=0.0, \mathrm{EPC}=1.0$ and $\mathrm{SC}=1.0$.

\section{Representing the validation scenario within the buildingEXODUS evacuation software}

A modified version of the buildingEXODUS [14], [15], [18] evacuation simulation software was used to simulate the validation data-set. As buildingEXODUS has been described in the literature many times this will not be repeated here, save to say that it is a rule based agent model operating on a fine nodal network. However, a brief description of the modifications to the software is presented. 
As part of the project it was noted that the walking speeds of workers is impacted by the nature of the temporary horizontal surface they were walking over (concrete, metal decking and rebar) and the type of temporary device they were travelling on between floors (dogleg or parallel scaffold stairs and ladders) [18]. To accurately simulate worker evacuation behaviour and performance on construction sites it is necessary to take this into consideration.

To achieve this the buildingEXODUS software was modified to include a capability to identify and differentiate between the different temporary floor surfaces (concrete, decking and rebar), an ability to represent an agents direction of travel on the decking (along the ridges or parallel to the ridges) and an ability to represent the different types of temporary vertical devices used for movement between floors (dogleg scaffold stairs, parallel scaffold stairs and ladders). In addition, data-sets to describe the performance of the workers over these various surfaces was collected and incorporated within the software. The full report details the data derived from the five small-scale walking speed trials involving 152 workers which support these observations [18], here we simply present a summary of the key findings.

The impact of different floor surfaces on travel speeds can be represented by a set of reduction factors applied to the agents walking speed on a normal flat surface. The reduction factor is also dependent on the length of time that the worker has worked on construction sites (i.e. their exposure experience) [18]. Table 1 presents the reduction factors appropriate for workers travelling across the different floor surfaces found in high-rise construction sites (concrete, metal decking and rebar).

Table 1. Walking speed reduction factors for different types of floor surfaces.

\begin{tabular}{|c|c|c|c|c|}
\hline Population & Concrete & Across decking & Rebar & Along decking \\
\hline Experienced & 1.0 & 0.80 & 0.78 & 0.73 \\
\hline Inexperienced & 1.0 & 0.76 & 0.72 & 0.68 \\
\hline
\end{tabular}

For vertical movement, the only temporary devices present during the full-scale evacuation trial used to define the HRCSVDS were scaffold dogleg stairs and ladders. From the small-scale walking speed trials, unobstructed descent speeds on temporary dogleg scaffold stairs varied from $0.42 \mathrm{~m} / \mathrm{s}$ to $1.21 \mathrm{~m} / \mathrm{s}$ with a mean of $0.72 \mathrm{~m} / \mathrm{s}$ while for ladders speeds varied from $0.29 \mathrm{~m} / \mathrm{s}$ to 0.61 $\mathrm{m} / \mathrm{s}$ with a mean of $0.45 \mathrm{~m} / \mathrm{s}$ [18]. Details of the walking speed distributions imposed on the simulations can be found in [18] and [19].

The geometry of the high-rise construction site was implemented within the modified software as shown in Fig. 5. This includes the specification of the temporary floor surfaces, scaffold stairs and ladders. Finally, the population, response times and specific evacuation procedure as described in Section 3 were also implemented within the software (see [19] for detailed specifications). 


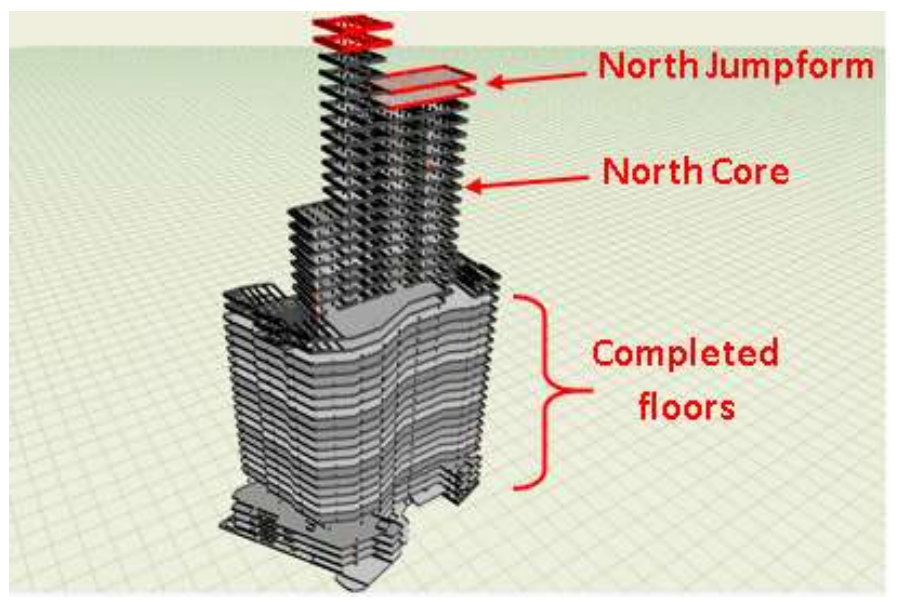

Fig. 5. The geometry of $22 \mathrm{BG}$ used in the validation case.

\section{Uncertainties in the HRCSVDS and sensitive analysis}

There are several uncertainties in the HRCSVDS which may impact the level of agreement between the model predictions and the experimental data. The complexity and layout of the internal clutter on most floors of the construction site was not recorded, and the precise initial location of the workers were unknown. How the simulation model is set up to accommodate these uncertainties will introduce a certain amount of variation in the model predictions. A series of sensitivity studies were conducted to explore the impact of the level of clutter in the jumpform and the starting floor location of the population on model predictions. As part of the sensitivity analysis all the other model parameters such as response time, are as specified within the HRCSVDS.

Firstly, the impact of the clutter on the evacuation time of workers in the jumpform is examined. Presented in Fig. 6 are the average exit curves (produced from 100 repeat simulations) for exiting from the jumpform and the main building for scenarios with and without clutter in the jumpform. The presence of clutter extends the overall average jumpform clearance time from $153 \mathrm{~s}$ to $160 \mathrm{~s}$, an increase of $7 \mathrm{~s}$ or about $5 \%$. However, there is no difference in building exiting times in both scenarios for the jumpform workers. The modest increase in time to exit the jumpform is expected as the clutter in the jumpform results in an increase in the average travel distance for agents to exit the jumpform. However, in terms of the building exit times, this modest increase in time to exit the jumpform is swamped by the more significant travel distances associated exiting the building as well as delays incurred by queuing when they interact with agents from other parts of the main building. Nevertheless, if clutter was represented within the model on each floor, it is expected that this would have a more significant impact on exiting times, shifting the predicted building exit curve towards longer exiting times. As the actual clutter present during the evacuation drill was not recorded and hence not included in the model (except for the jumpform), this will need to be taken into consideration when judging how close the model predictions are to the experimental data. 



Fig. 6. Average exit curves for scenarios with and without clutter in the jumpform.

391

392

393

394

395

396

397

398

399

400

401

402

\section{3}

Secondly, of the 190 workers in the main building, we know the starting floor for 63 workers, but not their precise starting location on the floor; and for 127 workers, their starting floor is only known to be within a range of floors. Thus, there is some degree of uncertainty in the precise starting location of the population. If these 127 workers were positioned on the lower floors within the ranges we would expect the predicted building exit curve (see Fig. 6) to be shifted to the left (towards lower exit times); while if they were located on the upper floors within the ranges, we would expect the predicted exit curve for the building population to be shifted to the right. As the starting location of the agents is not known, for each repeat simulation the starting locations are randomised within their known floor or a range of floors to examine the impact of the uncertainty in starting location on exiting time. Presented in Fig. 7 is the average exit time curve produced from 100 repeat simulations with the range of variation between the minimum and maximum values.

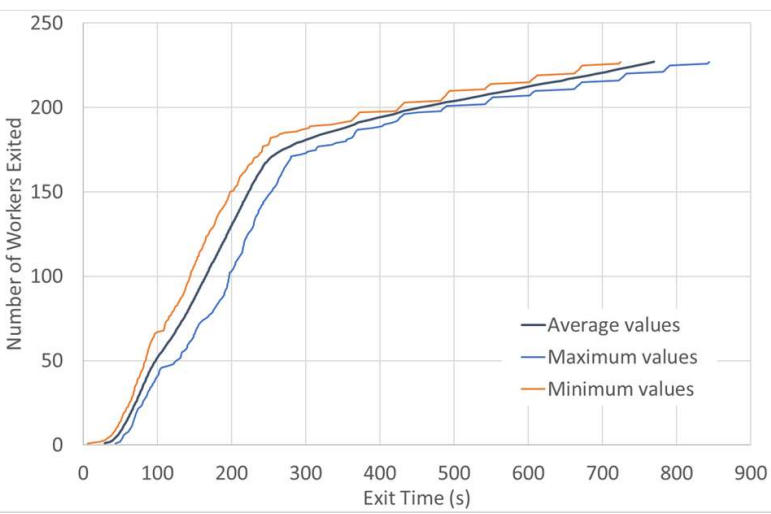

Fig. 7. buildingEXODUS predicted exit curves.

As can be seen, the maximum predicted exit time can be up to $58.2 \%$ greater than the minimum predicted evacuation times for the evacuation of the first 58 agents however, the percentage difference tends to diminish for the remainder of the population. As with the impact of clutter, this will need to be taken into consideration when judging how close the model predictions are to the experimental data. Furthermore, when comparing the predicted evacuation curve with the measured evacuation curve, the goodness of fit will depend on which particular simulation is selected for comparison purposes. Thus a means of selecting the appropriate curve is required. 


\section{Analysis of the evacuation software prediction using the performance metric}

Comparing the predicted average exit curve (see Fig. 7) with the experimental data, we note that the total evacuation time is over-predicted by $32 \mathrm{~s}$ or $4 \%$ ( $769 \mathrm{~s}$ compared with $737 \mathrm{~s}$ ), while the time for half of the population to exit the building is under-predicted by $52 \mathrm{~s}$ or $22 \%(180 \mathrm{~s}$ compared with $232 \mathrm{~s}$ ). The average time to clear the jumpform is under-predicted by $29 \mathrm{~s}$ or $15 \%$ (160 s compared with 189 s).

However, in order to assess how well the buildingEXODUS model prediction agrees with the experimental data, the three measures associated with the performance metric, i.e. ERD, EPC and $\mathrm{SC}$, are calculated for the 100 randomised simulations. The smallest and largest ERD values produced are 0.22 and 0.29 respectively. As the difference between the two values is small, it is suggested that all the simulations produce similar absolute differences between the predicted and measured data. The simulation producing the smallest ERD, i.e. 0.22 , is then selected as the simulation producing the best prediction of evacuation performance for the simulation model (see Fig. 8a). For this case, the difference between the predicted total evacuation time and the measured total evacuation time is an over-prediction of $60 \mathrm{~s}$ or $8 \%$ while the time for half the population to exit the building is under-predicted by $65 \mathrm{~s}$ or $28 \%$ ( $167 \mathrm{~s}$ compared with $232 \mathrm{~s}$ ). This simulation also produces an EPC value of 1.13. In order to determine the SC value an appropriate ratio $\mathrm{s} / \mathrm{n}$ must be selected to filter the noise out of the experimental data. The bumps in the experimental curve in Fig. 3 involves about 16 workers. To remove the influence of these bumps, an s/n of 0.07 is appropriate, i.e. for the 227 point data-set, the gradients used in the evaluation of Eq. 3 are spread over 16 data points. Based on this approach, the three measures of the performance metric for the buildingEXODUS simulation producing the smallest $\mathrm{ERD}$ are $\mathrm{ERD}=0.22, \mathrm{EPC}=1.13$ and $\mathrm{SC}=$ $0.82(\mathrm{~s} / \mathrm{n}=0.07)$.

As can be seen from Fig. 8a, the predicted exiting times are generally consistently smaller than the measured values, hence the relatively large value of ERD (0.22); however, the general shape of the predicted exit curve is a reasonable approximation to the experimental curve, hence the value of SC (0.82) being reasonably close to 1.0. The overall difference between the predicted and measured values could be further minimised if all the exit times could be increased slightly (hence the EPC value being larger than 1.0 and close to 1.0, i.e. 1.13). These results suggest that the buildingEXODUS model does a reasonable job in predicting the overall evacuation performance.

The performance metric can also be used to assess how well the predicted time to exit the jumpform matches the experimental data. Presented in Fig. $8 \mathrm{~b}$ is the predicted jumpform exit curve generated from the simulation that produced the smallest overall ERD. The ERD for the jumpform curve is 0.11 while the EPC value is 1.02 . 


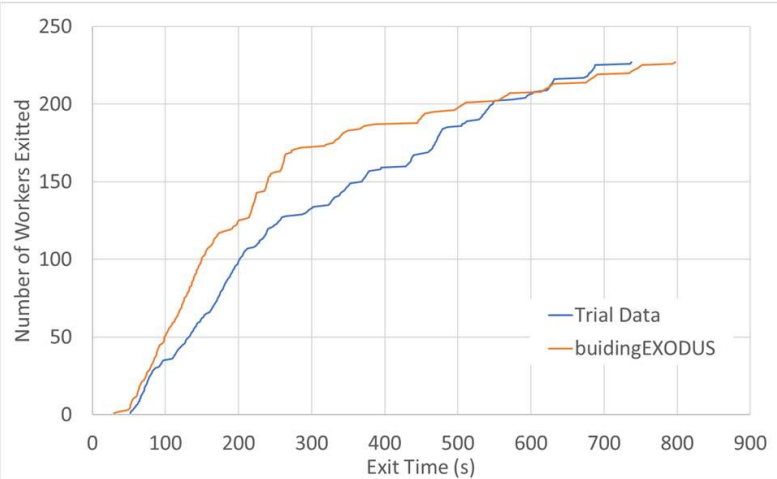

(a)

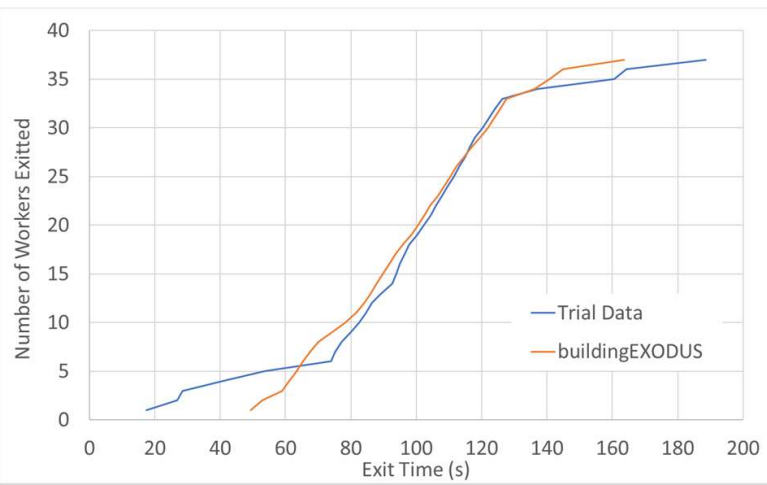

(b)

Fig. 8. The exit curves for the entire building and the jumpform with minimum overall ERD.

As previously, an appropriate $\mathrm{s} / \mathrm{n}$ ratio must be selected to filter the noise out of the experimental jumpform data. From the experimental curve presented in Fig. 4, the bumps in the curve involves 2 workers. To remove the influence of these bumps, an $\mathrm{s} / \mathrm{n}$ value of 0.05 would be appropriate, i.e. for the 37 point data-set, the gradients used in the evaluation of Eq. 3 are spread over 2 data points. Based on this approach, the three measures of the performance metric applied to the jumpform exiting data for the buildingEXODUS simulation producing the smallest overall ERD are $\mathrm{ERD}=0.11, \mathrm{EPC}=1.02, \mathrm{SC}=0.80(\mathrm{~s} / \mathrm{n}=0.05)$. For this case, the difference between the predicted time to clear the jumpform and the measured time is an under-prediction of $25 \mathrm{~s}$ or $13 \%$ (164 s compared with $189 \mathrm{~s}$ ).

As can be seen from Fig. 8b, in many places the predicted jumpform exiting times are almost identical to the measured values, hence the relatively small value of ERD (0.11), and the general shape of the predicted exit curve is a reasonable approximation to the experimental curve, hence the value of SC $(0.80)$ being reasonably close to 1.0 . The overall difference cannot be minimised by simply increasing or decreasing all the exit times as there are almost equal proportions of overprediction and under-prediction, hence the EPC value being 1.0. These results suggest that the buildingEXODUS model does a reasonable job in predicting the exiting time for the jumpform.

Given the uncertainties in the experimental data (uncertain starting location for some workers and a lack of representation of clutter on the floors), the level of agreement between the predicted and measured values produced by buildingEXODUS is considered acceptable and provides a means of specifying a benchmark defining an acceptable level of agreement with the experimental data (see Table 2). Thus, if other software tools produce a similar level of agreement for the HRCSVDS, then the software would be considered as capable as buildingEXODUS in predicting the outcome of this high-rise construction site evacuation trial.

The validation data-set outlined in this paper is described more thoroughly on the FSEG website [19]. The website provides details concerning the geometry (providing CAD DXF files), the initial population distribution, the specific evacuation procedure, the end-points for evaluation purposes, equations defining the population response time distributions and the arrival times for each worker at each end-point. Other parameters used in the simulations, such as population gender, age distribution, travel speeds, are also described. The website also suggests a systematic process for carrying out the validation assessment, which if followed, ensures consistency in the assessment process. A software tool is also provided to simplify the assessment of the performance metric. 
Table 2. HRCSVDS metric benchmark requirements.

\begin{tabular}{|l|l|}
\hline \multicolumn{1}{|c|}{ For overall predicted exit curve } & \multicolumn{1}{|c|}{ For predicted jumpform exit curve } \\
\hline (i) $\mathrm{ERD} \leq 0.22$ & (i) $\mathrm{ERD} \leq 0.11$ \\
\hline (ii) $0.87 \leq \mathrm{EPC} \leq 1.13$ & (ii) $0.98 \leq \mathrm{EPC} \leq 1.02$ \\
\hline (iii) $\mathrm{SC} \geq 0.82$ with $\mathrm{s} / \mathrm{n}=0.07$ & (iii) $\mathrm{SC} \geq 0.80$ with $\mathrm{s} / \mathrm{n}=0.05$ \\
\hline $\begin{array}{l}\text { (iv) Difference between the predicted and } \\
\text { measured total evacuation time for the } \\
\text { entire building to be within } 8 \% .\end{array}$ & $\begin{array}{l}\text { (iv) Difference between the predicted and } \\
\text { measured total exiting time for the } \\
\text { jumpform to be within } 13 \% .\end{array}$ \\
\hline
\end{tabular}

482

483

484

485

486

487

488

489

490

491

492

493

494

495

496

497

498

499

500

501

502

503

504

505

506

507

508

509

510

511

512

513

\section{Limitations}

As in any study there are limitations imposed on the findings due to practical constraints in collecting data and in performing the analysis. In interpreting the results presented in this paper, it is important to take these constraints into consideration.

There are several uncertainties in the HRCSVDS. Firstly, the complexity and layout of the internal clutter including obstacles and blockages on the floors of the 22 BG construction site were not recorded (except for the top deck of jumpform). Failing to represent the clutter on these floors may result in shorter predicted evacuation times compared with the actual experimental data. Secondly, the precise starting location of the construction site population is not known. This could have result in producing an over-prediction or under-prediction of the predicted evacuation times. Thirdly, the response time distribution imposed on the simulation is based on a generalised response time distribution from multiple trials, not just the data collected from the actual trial; thus, this may cause deviation from the actual evacuation performance.

The HRCSVDS includes data from a single evacuation trial and so the natural variation in evacuation behaviour cannot be represented. The authors welcome other researchers contributing additional high-rise construction site data to ensure that the proposed generalised relationships concerning response times and travel speeds are robust and to widen the scope of application to include other scenarios encountered in high-rise construction. Finally, the acceptance criteria for the performance metric are based on the performance of the modified buildingEXODUS evacuation software. Other models that produce similar simulation results that meet these criteria can be considered to produce predictions equivalent to those of buildingEXODUS for construction site evacuation.

\section{Conclusions}

While evacuation simulation tools can be used to develop, adapt and evaluate evacuation procedures for high-rise construction sites, for simulation models to be meaningful, they must be based on a reliable evidence base describing the performance and behaviour of construction workers during emergency evacuation situations. This data is essential to calibrate evacuation models thereby enabling them to be used for construction site applications. Furthermore, to improve the confidence in applying evacuation models to construction site applications, it is essential to have a reliable validation data-set representative of construction sites. 
In this paper a unique validation data-set for high-rise construction sites has been presented based on data generated from a series of unannounced evacuation trials conducted in high-rise construction sites. The data-set takes into consideration the impact of unique aspects of high-rise construction sites such as temporary floor surfaces, temporary scaffold stairs, ladders, the response of workers to the alarm and the two different physical environments associated with the main part of the building and the formworks. The use of the data-set, including an objective means of assessing performance was demonstrated using a modified version of the buildingEXODUS software. The assessment took into consideration uncertainties in the data-set associated with the precise starting location of workers and the presence of clutter on floors in the main building. The analysis has demonstrated that it is possible to predict the evacuation performance of high-rise construction sites with a reasonable level of accuracy if the simulation software is adapted to take into consideration the unique aspects associated with high-rise construction sites. Using such validated software it is hoped that the safety of high rise construction site workers will be enhanced through the development of more appropriate evacuation procedures.

\section{Acknowledgements}

The authors wish to thank Multiplex and the 1078 construction site workers who generously gave their time during the four evacuation trials and five walking speed experiments. The authors are also grateful to the Institution of Occupational Safety and Health (IOSH) for funding this work.

\section{References}

[1] NLA London Tall Buildings Survey 2019, New London Architecture (NLA), 2019, ISBN 978-1-9993513-0-4.

[2] Workplace fatal injuries in Great Britain 2018, Health and Safety Executive, July 2018.

[3] Fire safety in construction (2 ${ }^{\text {nd }}$ ed, 2010) HSG168, HSE Books 2010, ISBN9780717663453.

[4] Dylan Tutt, Andrew Dainty, Alistair Gibb, Sarah Pink, 2011, Migrant construction workers and health \& safety communication, Departments of Civil \& Building Engineering and Social Sciences, Loughborough University, ISBN 978-18575-1336-3.

[5] Galea, E. R., Deere, S. J., Hopkin, C. G., and Xie, H. (2017) Evacuation response behaviour of occupants in a large theatre during a live performance. Fire Material, Vol 41, issue 5, pp467 - 492, doi: 10.1002/fam.2424.

[6] Lovreglio R., Kuligowski E. Gwynne S., and Boyce K., A Pre-Evacuation Database for Use in Egress Simulations, Fire Safety Journal, Jan 2019, DOI: 10.1016/j.firesaf.2018.12.009

[7] CIBSE Guide E: Fire Safety Engineering, CIBSE, 2019, ISBN 9781912034291.

[8] H. Frantzich, Fire incidents during construction work of tunnels - evacuation aspects. Lund Report 3155, Lund 2010. ISSN: 1402-3504.

[9] Hisham Said, Amr Kandil and Hubo Cai, Agent-Based Simulation of Labour Emergency Evacuation in High-Rise Building Construction Sites, Construction Research Congress 2012: Construction Challenges in a Flat World. 2012, ASCE 2012, pp $1104-1113$, https://doi.org/10.1061/9780784412329.111 
[10] M. Abune'meh, R. El Meouche, I. Hijaze, A. Mebarki, I. Shahrour, Optimal construction site layout based on risk spatial variability, Automation in Construction, Volume 70, 2016, pp 167-177, https://dx.doi.org/10.1016/j.autcon.2016.06.014

[11] F Leite, Y Cho, A. H. Behzadan, S. H. Lee, S. Choe, Y. Fang, R. Akhavian and S. Hwang, Visulaization, Information Modeling, and Simulation Grand Challenges in the Construction Industry, 2016, 30(6), Journal of Computing in Civil Engineering, DOI: 10.1061/(ASCE)CP.1943-5487.0000604

[12] The Regulatory Reform (Fire Safety) Order 2005

[13] The Management of Health and Safety at Work Regulations 1999.

[14] Gwynne S, Galea E.R., Owen M., Lawrence P.J. and Filippidis L., A Review of the Methodologies Used in Evacuation Modelling, Fire and Materials, v23, 6, pp383-389, 1999.

[15] Wang Zhaozhi, Fuchen Jia, Galea Edwin R, and Choi Jun-Ho, 2017, A forensic analysis of a fatal fire in an indoor shooting range using coupled fire and evacuation modelling tools, Fire Safety Journal, Available online 6 April 2017, ISSN 0379-7112. http://doi.org/10.1016/j.firesaf.2017.03.029

[16] SFPE Guide to Human Behavior in Fire, 2019, Society of Fire Protection Engineers, Springer International Publishing, ISBN 978-3-319-94696-2

[17] Galea, E.R., Deere, S., Brown, R.,Filippidis, L., Two Evacuation Model Validation Datasets for Large Passenger Ships, SNAME (The Society of Naval Architects and Marine Engineers) Journal of Ship Research, Vol 57, number 3, pp155-170, Sept 2013, http://dx.doi.org/10.5957/JOSR.57.3.120037

[18] Galea, E.R., Deere, S., Xie, H., Hulse, L, Cooney, D., Construction Site Evacuation Safety Evacuation Strategies for Tall Construction Sites, report published by IOSH, Sept 2019. www.iosh.com/constructionevacuation

[19] Validation Document, https://fseg.gre.ac.uk/validation/building evacuation

[20] Peacock, R.D., Reneke, P.A., Davis, W.D., Jones, W.W.: Quantifying Fire Model Evaluation Using Functional Analysis, Fire Safety Journal, 22, 167-184, (1999).

[21] R. Lovreglio, E. Ronchi, D. Borri, 2014, The validation of evacuation simulation models through the analysis of behavioural uncertainty, Reliability Engineering \& System Safety, Volume 131, Nov. 2014, Pages 166-174. 